\title{
Direct Mapping of Massive Compact Objects in Extragalactic Dark Halos
}

\author{
Kaiki Taro Inoue \\ National Astronomical Observatory, Mitaka, Tokyo 181-8588, Japan \\ Masashi Chiba \\ Astronomical Institute, Tohoku University, Sendai 980-8578, Japan
}

\begin{abstract}
We propose a method for directly detecting MASsive Compact Objects (MASCOs) in extragalactic halos, using VLBI techniques with extremely high resolution $\sim 0.01 \mathrm{milli}$-arcsec. If a galactic halo comprising a large number of MASCOs produces multiple images of a background radio-loud QSO by gravitational lensing, then a high-resolution radio map of each macro-lensed image should reveal microlensing effects by MASCOs. Based on the size, position, and magnified or demagnified patterns of images, we shall be able to determine the mass and density profile of an individual MASCO as well as its spatial distribution and abundance in a galactic halo.
\end{abstract}

\section{Introduction}

There have been no stringent observational constraints on dark compact objects with a mass range of $10 M_{\odot} \leq M \leq 10^{4} M_{\odot}$. For brevity, we call them MASCOs (MASsive Compact Objects). Recent observations of the cosmic microwave background suggest that the primordial stars are rather massive as indicated by theoretical work, and their formation efficiency is higher than current theoretical work suggests. In some MASCO mass scales, primordial stars collapse directly to form black holes. Thus, a large fraction of baryonic dark matter in galactic dark halos may consist of black holes that are remnants of massive primordial stars.

\section{Method}

We consider a lensing system in which a radio-loud QSO is strongly lensed by the dark halo of an intervening galaxy. If MASCOs constitute a large amount of the dark matter component, then we expect to see many microlensing events by them, because the microlensing optical depth is nearly unity in a strong lens. In order to detect the signatures of such microlensing in the macro-lensed QSO images, one needs extremely high-resolution radio mapping of the images $(\sim 0.01$ milli-arcsec). Although current observations cannot achieve such a highresolution, it is not out of reach in the near future. 


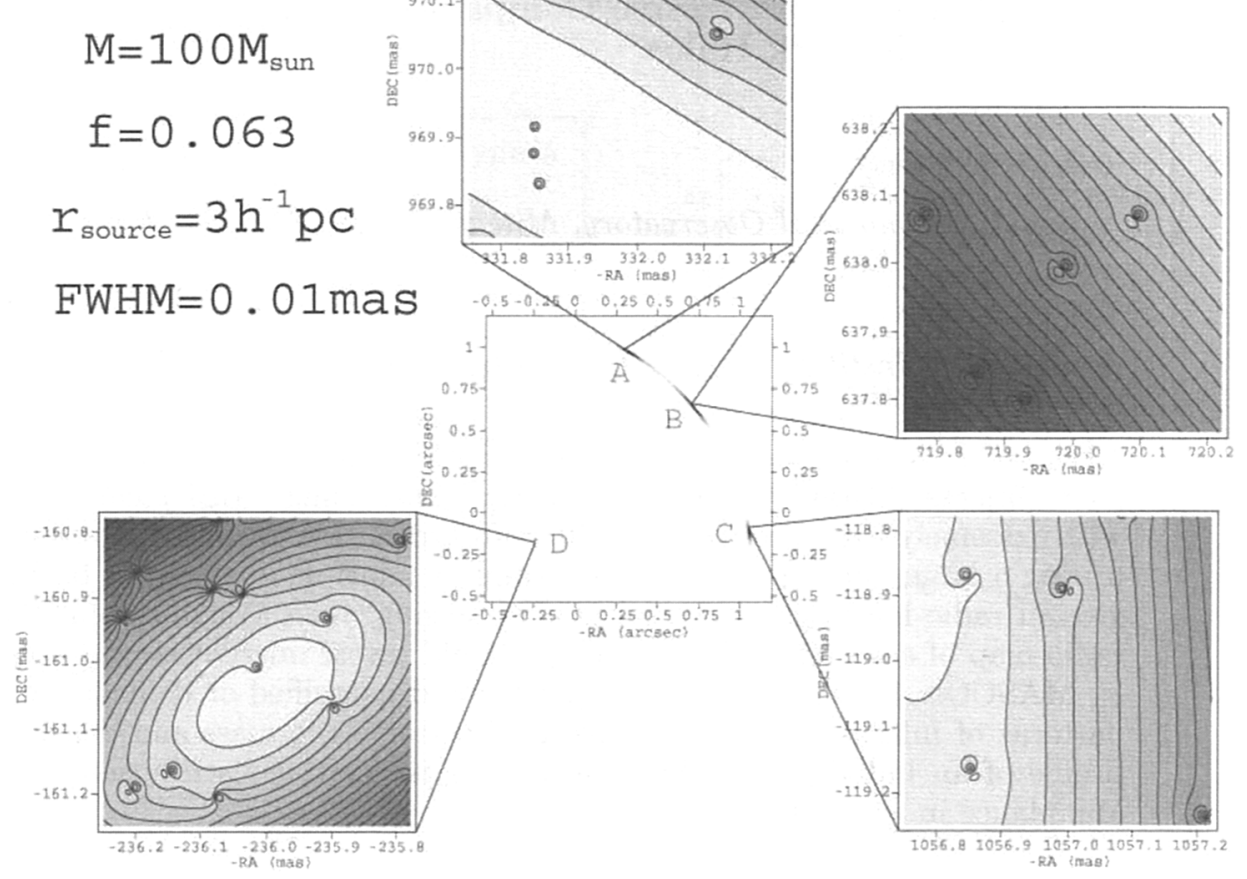

Figure 1. A simulation of radio maps of B1422+231 smoothed by a Gaussian beam with FWHM $=0.01$ mas. MASCOs are represented by point masses with mass $M=10^{2} M_{\odot}$. Dark spots in the 4 zoomed images correspond to MASCOs in the dark halo of the foreground galaxy. The isophote interval is $1 / 40$ of the maximum value. The surface brightness contrast between the dark and the bright spots is $1 / 10$ of the maximum value. The MASCO fraction to the dark matter halo is assumed to be $f=0.06$ corresponding to the MASCO density $\Omega_{*} \sim 0.018$ provided that the total matter density is $\Omega_{m}=0.3$.

\section{Result}

We present in Figure 1 numerical simulations for B1422+231, a typical strongly lensed QSO system. In the presence of MASCOs, tiny dark spots and pairs of dark and bright spots will appear in the macro-lensed images. From the size of the spot, one can estimate the mass of the MASCO. If a perturbing object of the macro-lensed image is described by a singular isothermal sphere (SIS), the signature of microlensing appears only if the gradient in the surface brightness is very large or the size of the SIS is sufficiently compact. In contrast to the pointmass case, light from the fringe of the source is not visible within the Einstein radius. Therefore, one can distinguish SISs from point masses by analysing the detailed spatial variation in the surface brightness. 